\title{
Molecular relaxation dynamics and self-association of dexamethasone sodium phosphate solutions
}

\author{
C. Kouderis ${ }^{1}$ - P. Siafarika ${ }^{1}$ - A. G. Kalampounias ${ }^{1,2}$
}

Received: 13 May 2021 / Accepted: 12 July 2021 / Published online: 19 July 2021

(c) Institute of Chemistry, Slovak Academy of Sciences 2021

\begin{abstract}
Detailed concentration-dependent measurements of sound absorption and velocity have been performed in dexamethasone sodium phosphate (DSP) aqueous solutions in the MHz frequency range. A single well-resolved relaxation process dominates the experimental acoustic spectra following a Debye-type distribution function. The analysis of the temperature-dependent ultrasonic relaxation data also revealed analogous effect with concentration on the relaxation spectra. All acoustic parameters were estimated by means of a fitting procedure. The behavior of the relaxation frequency and amplitude with concentration allowed us to assign the observed process to self-association mechanism. Combining the ultrasonic and electric conductivity data, the self-association scheme has been established. The thermodynamic constants and the rate of the aggregation due to hydrophobic interactions have been estimated in view of the Eyring's theory. The concentration dependence of relaxation amplitude and characteristic frequency revealed that the presence of additional relaxation processes in the spectra related to additional mechanisms, such as conformational changes and proton-transfer reaction is excluded and the self-association process considered here was found to dominate in this frequency range. The results have been discussed in view of the fair ability of DSP for hydrophobic interactions and aggregate formation in aqueous environment.
\end{abstract}

\section{Graphic abstract}
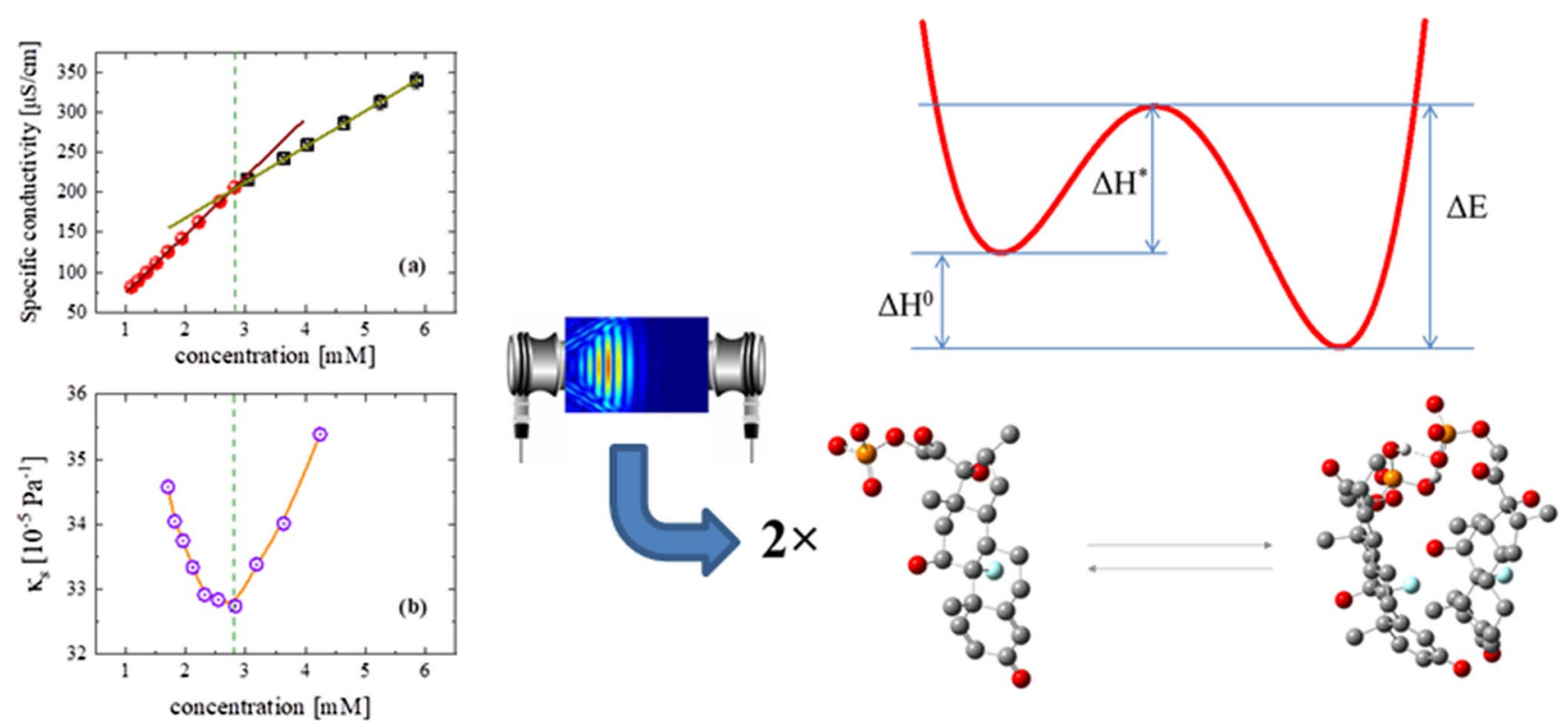

Keywords Ultrasonic relaxation · Self-association $\cdot$ Aggregation $\cdot$ Dexamethasone $\cdot$ Hydrophobic interactions

Extended author information available on the last page of the article 


\section{Introduction}

Dexamethasone sodium phosphate (DSP) is used in the treatment of emetic effects of cancer therapy and epicondylitis and it has anti-inflammatory action (Cassileth et al. 1983; Grunwald et al. 1984). On the other hand, dexamethasone is a synthetic pregnane corticosteroid and derivative of hydrocortisone (cortisol), while it is one of the most essential medicines of the World Health Organization's List (World Health Organization 2019). Dexamethasone belongs to the corticosteroids and is used for the treatment of rheumatic problems, several skin diseases, a variety of allergies, asthma and other lung diseases and brain swelling (National Institutes of Health 1995; Cohen et al. 2013). It is also used to improve outcomes in the baby after a preterm labor (National Institutes of Health 1995; Cohen et al. 2013). Cancer patients receiving undergoing chemotherapy are often given dexamethasone to counteract certain side effects of their antitumor treatments. It is also used as a direct chemotherapeutic agent or in combination with other chemotherapeutic drugs in certain hematological malignancies, especially in the treatment of multiple myeloma (Roila et al. 2000). Furthermore, in primary and metastatic brain tumors, dexamethasone can counteract the development of edema, which could sooner or later compress other structures of brain (Roila et al. 2000). Recently, dexamethasone is recommended by several institutions, such as the National Health Service in the UK and the National Institutes of Health (NIH) in the US, for patients with COVID-19 who need either mechanical ventilation or supplemental oxygen (without ventilation) (Sterne et al. 2020).

The molecular and crystal structure of dexamethasone and its stereochemistry have been determined in detail crystallographycally (Antignac et al. 2002; Raynor et al. 2007). This characteristic structure consisting of rigid aromatic fused rings exhibits a continuous association above a critical aggregation concentration (cac). Deep understanding of the DSP self-association and its thermodynamic profile is crucial for predicting the docking properties of this molecule, which has important implications in effective drug design. Ultrasonic relaxation spectroscopy has been proved as an effective tool in studying associationdissociation mechanisms (Nishikawa et al. 1991), protontransfer reactions (Nishikawa et al. 1973a, b, 1996; Nishikawa and Kamimura 2011; Huang et al. 1999; Tsigoias et al. 2020a), conformational changes (Bae et al. 2001; Bae et al. 2002; Kalampounias 2020; Stogiannidis et al. 2020), complexation (Tamm 1961; Petrucci 1971), backbone segmental motion and normal mode relaxation in polymers (Kouderis et al. 2021; Siafarika et al. 2021) and reaction engineering in general (Bernasconi 1976). Furthermore, in several cases systematic analysis of the acoustic spectra permits disentangling between different processes taking place in close time scales (Kouderis et al. 2021).

In this work, we conducted a comprehensive temperatureand concentration-dependent study by means of ultrasonic relaxation spectroscopy of aqueous DSP solutions in an effort to comprehend on the molecular relaxation dynamics. The acoustic spectra have been used to expose the relaxation process and clarify the underlying processes occurring in the system suggesting the possibility of a self-association scheme. The thermodynamic parameters of the self-association have also been determined. Computational methodologies have been utilized to evaluate the properties of the isolated molecule and aggregate in gaseous state where no interactions are present and in solvation state with water as solvent. Furthermore, the thermodynamic characteristics of the self-association have been estimated in an effort to elucidate the proposed mechanism.

\section{Experimental procedure}

\section{Solutions}

Dexamethasone sodium phosphate (percent purity 98.0\%, Alfa Aesar) is a white hygroscopic powder, incompatible with oxidizing agents and it has molecular formula $\mathrm{C}_{22} \mathrm{H}_{28} \mathrm{FNa}_{2} \mathrm{O}_{8} \mathrm{P}$. DSP was used as received to prepare solutions with the desired concentration ranging from 1.71 to $6.45 \mathrm{mM}$. Crystalline solid was dissolved to triply distilled water at ambient pressure and temperature. The final solutions were clear and colorless. Only fresh solutions were used for conducting the complete set of measurements.

\section{Measurement principles of sound absorption and velocity}

The measurement setup utilized for the simultaneous detection of sound absorption and velocity has been described in (Mpourazanis et al. 2019; Kalampounias 2020; Stogiannidis et al. 2020). The apparatus consists of two identical broad band piezoelectric transducers (Olympus, nominal center frequency $10 \mathrm{MHz}$ ) and a cylindrical thermostated acoustic cell with parallel phases. The ultrasonic transducers are mounted on opposite parallel sides of the sample container serving as signal transmitter and detector of the transmitted signal after traveling through the sample. The length of the acoustic path is fixed at $L=1 \mathrm{~cm}$. The transducer diameter is $0.5 \mathrm{inch}$. An RF signal generator (TTi, Model: TGR 1040) is utilized for the excitation of the transducer by a sinusoidal burst consisting of 1 cycle with a $5 \mathrm{~ms}$ repetition-rate and frequency ranging between 1 and $50 \mathrm{MHz}$. The output signal is sent to a digital oscilloscope (TEKTRONIX, Model: 
TBS1152B) for monitoring the signal in the time-domain. The excitation signal from the signal generator is used to trigger the oscilloscope.

In an effort to determine experimentally the excess absorption coefficient $\alpha_{\text {excess }}(f)$ as a function of frequency, one should measure the transmitted wave through solvent (water) $S_{\text {solvent }}(t)$ and the transmitting wave through solution $S_{\text {solution }}(t)$ for a specific ultrasound frequency in the timedomain. Then, the excess absorption coefficient $\alpha_{\text {excess }}(f)$ is estimated as a function of frequency in $\mathrm{Np} / \mathrm{cm}$ through the equation:

$a_{\text {excess }}(f)=\frac{1}{L} \ln \left\{\frac{\left|\operatorname{FFT}\left(S_{\text {solution }}(t)\right)\right|}{\left|\operatorname{FFT}\left(S_{\text {solvent }}(t)\right)\right|}\right\}$

The excess absorption coefficient $\alpha_{\text {excess }}(f)$ is the absorption of the solution after subtracting the absorption of the solvent for the specific ultrasound frequency and $L$ is the length of the acoustic travelling path that was fixed for the acoustic cell used in this study. In our case, the solvent absorption is constant in the frequencies covered here exhibiting a non-relaxing behavior. FFT denotes the Fast Fourier Transform of the corresponding signals after appropriate zero padding. The experimental error in sound absorption measurements is $\pm 5 \%$.

The sound velocity is estimated for all concentrations studied by determining the time required for the ultrasound to travel the fixed path length (Mpourazanis et al. 2019; Kouderis et al. 2021). The experimental error in velocity measurements is $\pm 0.01 \%$. It is expected velocity to exhibit dispersion with frequency. For this reason, velocity has been measured in a wide frequency range. The velocity dispersion with frequency is not experimentally observed when the criterion $\frac{\alpha u_{0}}{2 \pi f} \ll 1$ is satisfied (Lee et al. 2017). For the lowest and highest concentrations studied, the ratio was calculated between $6.20 \times 10^{-4}$ and $2.62 \times 10^{-3}$ that is far below unity and velocity dispersion is not likely to arise in the frequencies covered here. Indeed, our experimental velocities were found frequency independent within experimental error. In the following sections, we present only the experimental values corresponding to $10 \mathrm{MHz}$. The measurements of the acoustic parameters have been performed in the $16-36{ }^{\circ} \mathrm{C}$ temperature range within $\pm 0.01{ }^{\circ} \mathrm{C}$.

The experimental setup developed in our laboratory is appropriate for measuring both sound absorption and speed in liquids and solutions at selected temperature under constant pressure conditions. For validation and calibration reasons, we measured the sound absorption and velocity of several liquids and pure solvents (Stogiannidis et al. 2020). The satisfying agreement between our experimental and literature values reveals that our methodology is reliable for simultaneous measurements of sound absorption and sound speed in liquids.

\section{Conductivity and density measurements}

Experimental densities of the solutions were measured by means of a density-measuring cell (Anton Paar, DMA 60). The uncertainty of the measurements was $\pm 0.0001 \mathrm{~g} / \mathrm{cm}^{3}$. Conductivity was estimated using conductivity meter (Orion Research, Model 101) allowing temperature compensation, $\%$ temperature coefficient settings, cell constant and ranges adjustment, backing off potentiometer and frequency selection with accuracy better than $1 \%$ of the full scale. Both density and conductivity measurements were thermostated at the desired temperature using a water circulator.

\section{Theoretical calculations}

Quantum-mechanical calculations for dexamethasone sodium phosphate and for the system resulting from the aggregation of two dexamethasone sodium phosphate molecules were carried out with the Gaussian 09 W Revision D.01 package of programs (Lee et al. 1988; Becke 1993; Frisch et al. 2013). Density functional theory (DFT) using Becke's three parameter exchange functional with the Lee-Yang-Parr correlation functional (B3LYP) and 6-31G(d) basis set was used for structure optimization and molar volume estimation under tight optimization convergence criteria (Lee et al. 1988; Becke 1993; Frisch et al. 2013). The calculations of the reaction volume change $\Delta V$ were performed both in the gaseous phase and in the presence of water solvent. Molecular volume was estimated theoretically by considering the space included in a contour of a particular electron density (0.001electrons/Bohr $\left.{ }^{3}\right)$. The volume of each atom existing in the molecule was estimated by performing a quantum calculation in order to receive the corresponding wavefunction, which is further integrated to obtain volume. The sum of all individual volumes provides the molecular one. The 6-31G(d) is a split-valance basis set which includes also $d$-type polarization functions. The structure of dexamethasone sodium phosphate was obtained from the Pubchem electronic database as SDF file. The study of the aggregation was performed in AutoDock Tools-1.5.6 software. The optimized geometries of the structures after minimizing energy emerged from AutoDockTools-1.5.6 program with respect to the coordinates of atoms and no restrictions on symmetry. A grid box was defined containing all potential interacting units. Molecular interaction study of each aggregation species was performed and the corresponding energies of the various poses during their interaction were received from the calculation. These interactions and bond distances for their best pose were analyzed utilizing Pymol software. Following the typical molecular docking 
procedure, for each calculation 9 total poses were retained after clustering and subsequently the most stable single pose was received corresponding to binding energy of a few kcal/ mol. Enthalpy difference was estimated by means of semiempirical methods to reduce calculation cost, which is relative excessive for a chemical system as DSP dimer, which is composed of 124 atoms including phosphate, $-\mathrm{OH},-\mathrm{F}$ and $\mathrm{C}=\mathrm{O}$ functional groups. More specifically, the popular MNDO-type PM6 D3 method was used for the calculation of the enthalpy difference related to the dimerization reaction.

\section{Results and discussion}

\section{Dynamic and structural processes in DSP aqueous solutions}

The molecular structure of dexamethasone sodium phosphate after optimization is shown in Fig. 1a. The structure of dexamethasone is also shown in Fig. $1 \mathrm{~b}$ for comparison. Close examination of the DSP structure reveals that it is dominated by the presence of fused aromatic rings, the phosphate group and additional functional groups, such as $-\mathrm{OH}$, $-\mathrm{F}, \mathrm{C}=\mathrm{O}$. It seems that the specific structure may demonstrate not only a molecular self-aggregation reaction, but also conformational changes. Additional processes, such as a proton-transfer reaction of DSP with the solvent may also be present.

The self-aggregation reaction of DSP is described as:

$n D S P \rightleftarrows(\mathrm{DSP})_{n}$

The above equation considers the usual stepwise aggregation reaction as a mean mechanism, which is valid for low $n$ values, where $n$ is the aggregation number and (DSP) $)_{n}$ is the aggregate. At intermediate concentrations of DSP aqueous solutions, the solute-solvent interactions are stronger and an association scheme with mixed water and DSP molecules is also possible. This interaction is given by:

$\left(\mathrm{H}_{2} \mathrm{O}\right)_{m}+\mathrm{DSP} \rightleftarrows \mathrm{DSP} \cdot\left(\mathrm{H}_{2} \mathrm{O}\right)_{m}$

Complexes $\left(\mathrm{H}_{2} \mathrm{O}\right)_{m}$ and DSP $\cdot\left(\mathrm{H}_{2} \mathrm{O}\right)_{m}$ represent the water aggregate and the mixed aggregate of water and DSP, respectively. Both complexes are formed by hydrogen-bonding.

The equilibrium between different conformers may be presented as:

$\mathrm{DSP} \rightleftarrows \mathrm{DSP}^{*}$

where DSP* represents a possible conformer of DSP.

Finally, the proton-transfer reaction is generally described as:

$\mathrm{DSP}+\mathrm{H}_{2} \mathrm{O} \rightleftarrows \mathrm{DSPH}^{+} \ldots \mathrm{OH}^{-} \rightleftarrows \mathrm{DSPH}^{+}+\mathrm{OH}^{-}$

\section{Analysis of the relaxation behavior-Concentration effect}

The dynamic and structural properties of DSP aqueous solutions can be clarified by ultrasonic relaxation spectroscopy. In Fig. 2 are presented the excess experimental $\alpha / f^{2}$ values and the corresponding fitting curves as a function of frequency for all concentrations studied. The solvent absorption coefficient is frequency independent in the $\mathrm{MHz}$ range and is subtracted from the absorption coefficient value of the solution providing thus the excess $\alpha / f^{2}$ values.

Solid lines correspond to individual relaxation curves modeled by Debye-type equation:

$$
\frac{a}{f^{2}}=\frac{A}{1+\left(\frac{f}{f_{r}}\right)^{2}}+B
$$

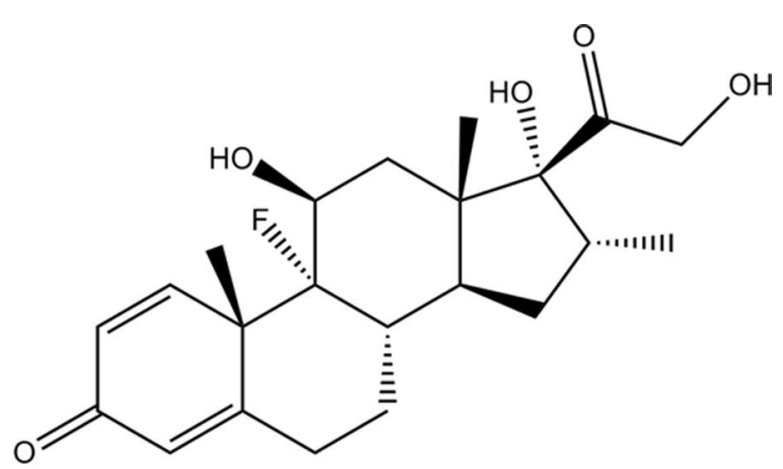

(a)

(b)

Fig. 1 Representations of molecular structure of a Dexamethasone sodium phosphate (DSP) and b Dexamethasone molecule 


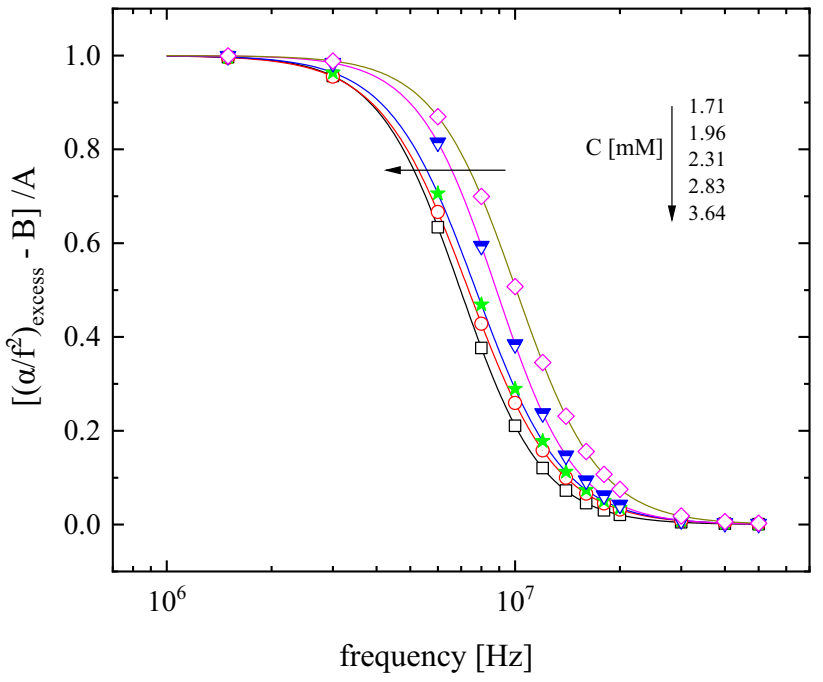

Fig. 2 Normalized excess ultrasonic absorption values versus ultrasound frequency for all concentrations studied. Symbols represent experimental values, while lines denote the corresponding curves after the fitting procedure. The constant and non-relaxing ultrasonic absorption of the solvent (water) was evaluated equal to $2.2 \times 10^{-16} \mathrm{~s}^{2} /$ $\mathrm{cm}$ and is subtracted from the ultrasonic absorption of solutions

where $f_{r}, A$ and $B$ is the characteristic relaxation frequency, the relaxation amplitude and the background absorption, respectively. The characteristic frequency is defined as the sagmatic point of the sigmoidal function in the frequencydomain. In order to stress out the frequency shift of the curves, the $y$-axis is zero to one normalized through the equation $\left(\left(\frac{\alpha}{f^{2}}\right)_{\text {excess }}-B\right) / A$. The spectra of Fig. 2 evidently expose the presence of only one relaxation process with its characteristic frequency shifting to lower frequencies with increasing concentration. The presence of an additional relaxation process would be observed as a second sagmatic point in the sigmoidal function with lower or higher characteristic frequency. The relaxation frequency variation with increasing solution concentration indicates that the mechanism behind the observed relaxation process involves cooperative motion of molecule assembles.

As stated above, at least three distinct mechanisms (aggregation, conformational changes, proton-transfer) are expected in the acoustic spectra of DSP aqueous solutions. Nevertheless, only one relaxation mechanism is evident in the recorded spectra, which lies in the $7-11 \mathrm{MHz}$ frequency region. In order to elucidate the origin of the observed mechanism, we examined the concentration dependent of the acoustic parameters as obtained from the iterative fitting procedure based on the nonlinear regression Levenberg-Marquardt algorithm (Kalampounias et al. 2013). The validation of the fitting using methodologies other than Levenberg-Marquardt's method did not provide better goodness of fit. The outcome of the analysis may lead us to

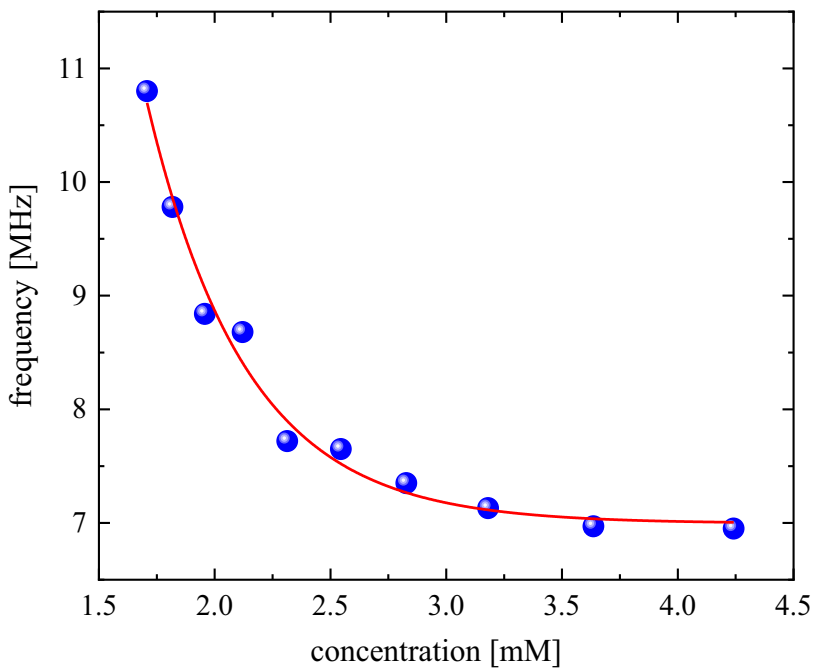

Fig. 3 Concentration dependence of the characteristic relaxation frequency for all concentrations studied at ambient conditions. Symbols represent experimental values and line is used as a visual guide

draw conclusions concerning the assignment of the observed relaxation mechanism. Indeed, the relaxation frequency and relaxation amplitude seem to follow a rather non-monotonic variation with concentration as observed in Figs. 3 and 4, respectively. If a conformational change was attributed to the observed relaxation in the spectra, then the relaxation frequency would be independent of concentration and the relaxation amplitude would linearly increase with increasing DSP concentration, as it has been recently reported for methyl acetate-ethanol solutions (Stogiannidis et al. 2020) and pure n-propyl formate liquid (Kalampounias 2020). Thus, the assignment of the present relaxation to conformational changes is excluded. Furthermore, both $f_{r}$ and $A$ fitting parameters seem to follow different trends below and above $\sim 2.82 \mathrm{mM}$, which corresponds to $x=0.08 \mathrm{~mol}$ fraction. On the other hand, if the observed relaxation was attributed to proton-transfer reaction, the characteristic frequency and relaxation amplitude would follow a rather monotonic increase with solution concentration, as observed in the case of aqueous polyamine solutions (Siafarika et al. 2021) and aqueous poly-vinyl-alcohol solutions (Kouderis et al. 2021). Thus, the assignment of the present relaxation to protontransfer reaction is questionable. Furthermore, a relaxation attributed to proton-transfer mechanism would be expected in a frequency range much higher than that observed here, since the proton exchange process is much faster than the making-breaking of hydrogen-bonds, which is probably the dominating mechanism. All these arguments led us to the conclusion that the dominant interactions taking place in this system are mainly intermolecular, such as the aggregation reaction. The theoretical molecular interaction study of the aggregation species revealed the formation of a stable 
Fig. 4 Left axis: Concentration dependence of the relaxation amplitude as received from the fitting procedure for all concentrations studied. Right axis: Ultrasound velocity as a function of concentration for the same solutions. Symbols represent experimental values and curves correspond to polynomial fittings and are used as guide to the eye. A fourorder polynomial has been used for relaxation amplitude data $\left(R^{2}=0.946\right)$ and a third-order polynomial has been used for sound velocity data $\left(R^{2}=0.991\right)$

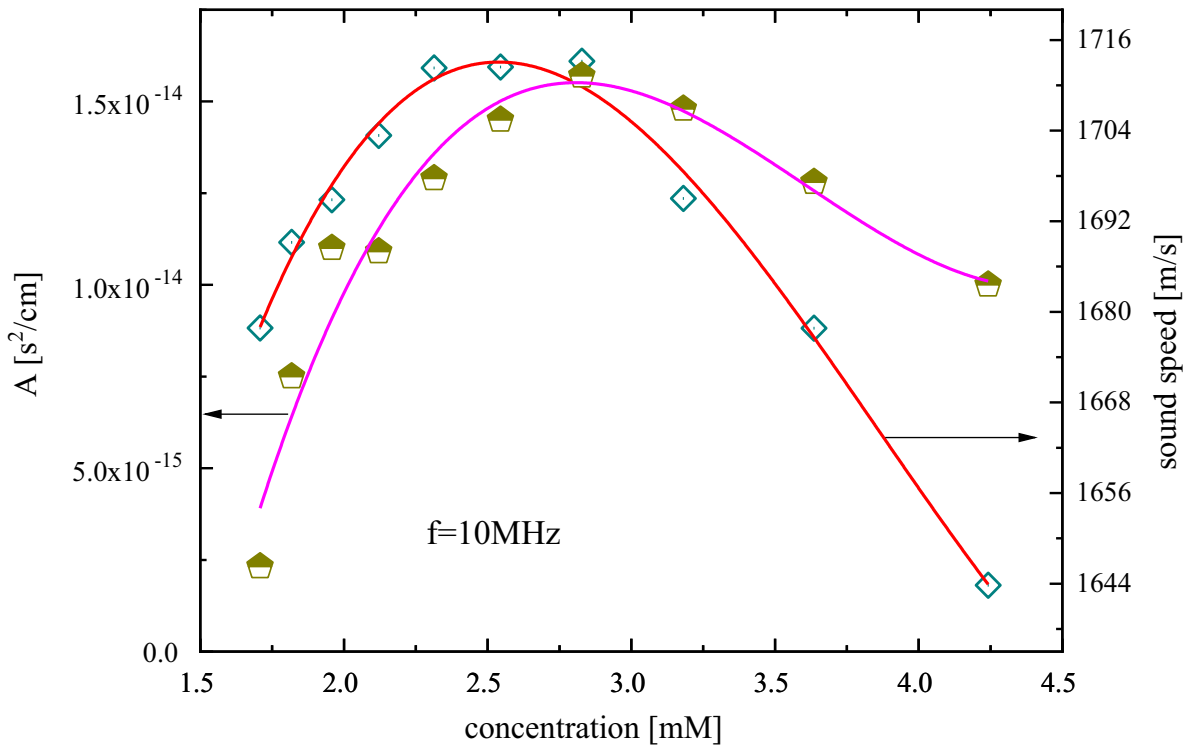

aggregate composed of two monomers with binding energy of $2.91 \mathrm{kcal} / \mathrm{mol}$. The transition to a more compact structure with aggregates should be reflected in the sound velocity variation with concentration. Indeed, the sound speed versus DSP concentration exhibits a characteristic non-monotonous trend with a break near $\sim 2.55 \mathrm{mM}$.

The maxima observed in the relaxation amplitude or in sound speed plots versus solution concentration are a universal characteristic that has been noticed in the past in binary and ternary aqueous alcoholic (Verall et al. 1977 and references therein) and amine (Barfield and Schneider 1959; Tsigoias et al. 2020, 2021) solutions that are typical hydrogen-bonding systems. The concentration corresponding to the peak, the so-called peak sound absorption concentration (P.S.A.C.), is frequency dependent. If the excess absorption mechanism in DSP aqueous solutions was due to association scheme expressed by Eq. (2), then the PSAC would be expected near $0.5 \mathrm{~mol}$ fraction of the DSP. Though, the peak concentration is observed at $2.82 \mathrm{mM}$, which corresponds to $x=0.08 \mathrm{~mol}$ fraction. Therefore, the scheme designated by Eq. (2) must be ruled out and the cause of the excess absorption is the mechanism presented in Eq. (1).

The concentration dependence of the relaxation amplitude (excess absorption) and sound velocity is presented in Fig. 4 allowing direct comparison. It seems that the concentration where both quantities exhibit their maxima is somewhat different. This experimental finding has been observed in the past in other binary systems (see e.g. Tsigoias et al. 2020). The experimental determination of the specific conductivity of the DSP solutions may provide further insights into association mechanism by estimating the critical aggregation concentration (cac), which can be determined as the cross point of two straight lines passing through the specific conductivity data below and above this characteristic concentration. In order to increase the precision in the estimation of cac, a large number of experimental points in the specific concentration range is necessary. Indeed, the specific conductivities of a large amount of DSP solutions were measured and the results are presented in Fig. 5a. A typical for aggregation mechanism variation of conductivity is observed below and above cac. The data for lower and higher concentrations than cac were linearly fitted and the cross point is observed at $2.82 \mathrm{mM}$, which perfectly matches the PSAC value. In addition, the adiabatic compressibility values as a function of concentration for the same solutions were calculated and the results are presented in Fig. 5b. Adiabatic compressibility exhibits a minimum and can also be used to determine analogous structural effects. By comparing the specific conductivity and adiabatic compressibility data, a coincidence between their respective characteristic points (cross point and minimum, respectively) is evident further supporting our assumption that the dominating process is the molecular self-association of DSP and not a relaxation process due to internal vibrational modes.

It is more than clear that hydrogen-bonding between molecules is the driving force behind this relaxation. Formation and braking of hydrogen-bonds causes a perturbation in the propagation of the acoustic waves with a parallel total volume change and thus a compressional relaxation. A quantitative description of a mechanism behind a relaxation process like this one, cannot be easily provided due difficulties related to the inherent intermolecular complexity of hydrogen-bonded aqueous solutions. Considering that Eq. (1) describes the above mechanism in one mean stage, we can express the relation between the relaxation frequency $f_{r}$ in $\mathrm{MHz}$ and the reactant concentration [DSP] as (Nishikawa et al. 1991; Nishikawa and Kamimura 2011): 

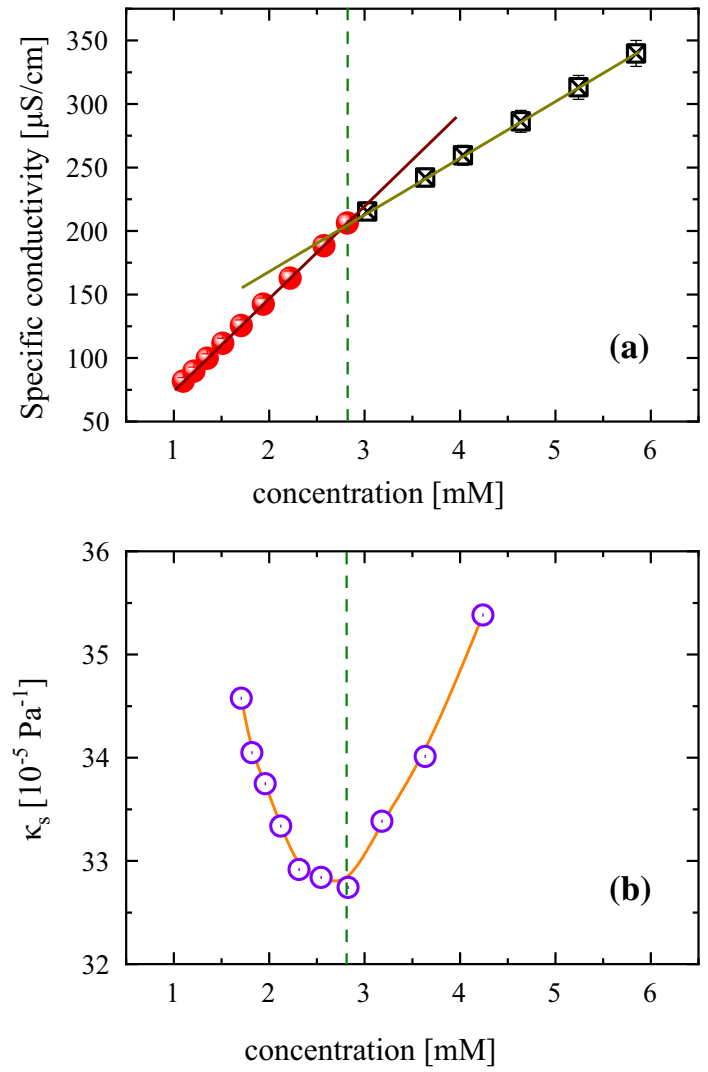

Fig. 5 a Plot of specific conductivity versus solution concentration. Solid lines represent linear fittings. The critical aggregation concentration (cac) corresponds to the inflection point, which can be determined as the intersection of the two linear fits. $\mathbf{b}$ Adiabatic compressibility (open symbols) as a function of concentration for the same solutions calculated as $\kappa_{s}=\left(\rho \cdot u_{s}^{2}\right)^{-1}$. Solid line represents polynomial fit and is used as a visual guide. Note the coincidence between specific conductivity and adiabatic compressibility inflection points

$2 \pi f_{r}=n^{2} k_{f}[\mathrm{DSP}]^{n-1}+k_{b}$

where $n$ is the aggregation number, while constants $k_{f}$ and $k_{b}$ represent the forward and backward rate constants, respectively. Several values on $n$ were tested in order to receive the least error of plots of $2 \pi f_{r}$ vs $[\mathrm{DSP}]^{n-1}$. Values higher than 2 provided continuously increasing errors and thus the most favorable value of the aggregation number is equal to $n=2$. Subsequently, the values of the forward and backward rate constants were found equal to $k_{f}=6.65 \times 10^{7} \mathrm{M}^{-1} \mathrm{~s}^{-1}$ and $k_{b}=4.52 \times 10^{7} \mathrm{~s}^{-1}$. The main outcome from the theoretical calculations performed is that dimer species reveals as the most thermodynamically favorable. From the experimental values of relaxation frequency, maximum excess absorption per wave length and forward constant, the volume change of the reaction can be evaluated from the following equation (Nishikawa et al. 1973b): $\mu_{\max }=\frac{\pi \rho u^{2}}{2} \frac{(\Delta V)^{2}}{R T} \Gamma$

where $\mu_{\max }=\frac{1}{2} \operatorname{Auf} f_{r}$ is the maximum value of the absorption per wavelength at the relaxation frequency $f_{r} . \Delta V$ is the standard volume change given by $\Delta V=V_{2}-n V_{1}$ with $V_{l}$ and $V_{2}$ designating the partial molar volumes of DSP monomer and (DSP) ${ }_{n}$ aggregate, respectively. $R, T, \rho$ and $u$ correspond to the gas constant, absolute temperature, solution density and sound velocity, respectively. Finally, $\Gamma$ is the concentration term given by (Kaatze et al. 2000):

$\Gamma^{-1}=\sum_{j} \frac{\Delta v_{j}^{2}}{c_{j}}-\frac{1}{c}\left(\sum_{j} \Delta v_{j}\right)^{2}$

where $c_{j}$ are the molar concentrations corresponding to equilibrium state and $\Delta \nu_{j}$ is the difference of the stoichiometric coefficients of the reactants and products. Parameter $j$ designates the number of the species involved in the studied reaction.

Using the experimental values of the parameters involved in Eq. (7), the volume change of the reaction has been estimated as $\Delta V=(30.6 \pm 1.5) \times 10^{-6} \mathrm{~m}^{3} / \mathrm{mol}$. The theoretically calculated values of volume change in the vapor state is $\Delta V_{t h}=49.3 \times 10^{-6} \mathrm{~m}^{3} / \mathrm{mol}$, while in solution state with water is $\Delta V_{t h, \mathrm{H} 2 \mathrm{O}}=43.3 \times 10^{-6} \mathrm{~m}^{3} / \mathrm{mol}$. The observed discrepancy in the experimental and theoretical values are expected since in the theoretical calculation intermolecular interactions between neighboring species where not taken into account in the calculation. It is interesting to note that the theoretical value of the volume change is closer to the experimental $\Delta V$ when solvent effect is considered in the calculation. Nevertheless, the comparison between the experimental data and the outcome of the theoretical calculations evidenced that the present mechanism is related with the aggregation scheme proposed. Volume changes associated with conformational changes would be negligible.

\section{Analysis of the relaxation behavior-Temperature effect}

In an effort to examine the thermodynamic characteristics of the aggregation mechanism, we measured the acoustic spectra as a function of temperature for a DSP aqueous solution with concentration $3.87 \mathrm{mM}$, which is above the critical aggregation concentration and representative results are presented in Fig. 6 for three selected temperatures.

It seems that temperature affects significantly the acoustic spectra and the characteristic relaxation frequency exhibits a blue shift with increasing temperature. The relaxation frequency is associated to the kinetic parameters of relaxation in the context of the Eyring's theory as (Herzfeld and Litovitz 1959; Blandamer 1973; Ensminger et al. 2011): 


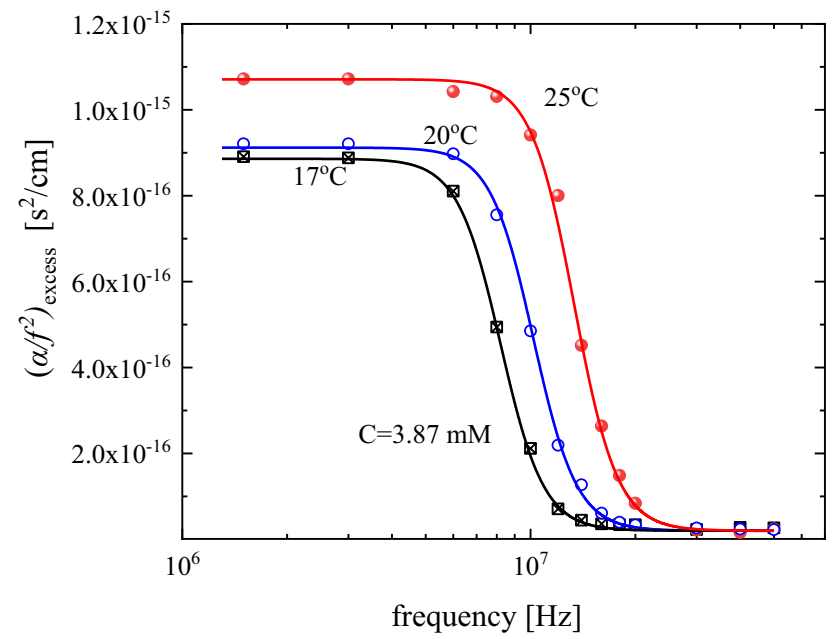

Fig. 6 Temperature dependence of the excess ultrasonic absorption values of DSP aqueous solution with concentration $C=3.87 \mathrm{mM}$. Symbols represent experimental values and curves correspond to Debye-type fittings

$\tau=\frac{h}{k_{B} \mathrm{~T}} \exp \left(\frac{\Delta G^{*}}{R T}\right)$

$\Delta G^{*}$ is the activation free energy given by $\Delta G^{*}=\Delta H^{*}-T \cdot \Delta S^{*}$ and $\tau=\left(2 \pi \cdot f_{r}\right)^{-1}$ the relaxation time. Constants $k_{B}$ and $h$ have their usual meanings, namely the Boltzmann and Planck constants. The above equation can be written in linear form as:

$\ln \left(\frac{f_{r}}{T}\right)=-\frac{\Delta \mathrm{H}^{*}}{1000 R}\left(\frac{1000}{T}\right)+\left[\frac{\Delta S^{*}}{R}+\ln \left(\frac{k_{B}}{2 \pi h}\right)\right]$

Parameters $\Delta H^{*}$ and $\Delta S^{*}$ are the activation enthalpy and the corresponding activation entropy, respectively. By plotting the ratio $\ln \left(f_{r} / T\right)$ as a function of reciprocal temperature, one is able to determine the activation enthalpy from the slope and the activation entropy from the intercept of the graph. The $\ln \left(f_{r} / T\right)$ versus $1 / T$ dependency is presented in Fig. 7 for all temperatures studied revealing a clear linear behavior. The activation enthalpy, estimated from the slope, was found equal to $\Delta H^{*}=4.23 \pm 0.34 \mathrm{kcal} / \mathrm{mol}$, while the corresponding activation entropy was determined from the intercept equal to $\Delta S^{*}=-20.1 \pm 1.6 \mathrm{cal} / \mathrm{molK}$. It seems that the $\Delta S^{*}$ contribution to the change of free energy is insignificant, indeed $\Delta S^{*} / \Delta H^{*} \approx 0.48 \%$.

The relaxation strength is given by (Lamb 1965):

$r=\frac{A u f_{r}}{\pi}-\frac{1}{4}\left(1-\frac{2 \mathrm{~B}}{\mathrm{~A}}\right)\left(\frac{A u f_{r}}{\pi}\right)^{2}$

All parameters appearing in the above equation have been evaluated experimentally and the resulting relaxation strengths varies between 0.00134 and 0.00629 for the

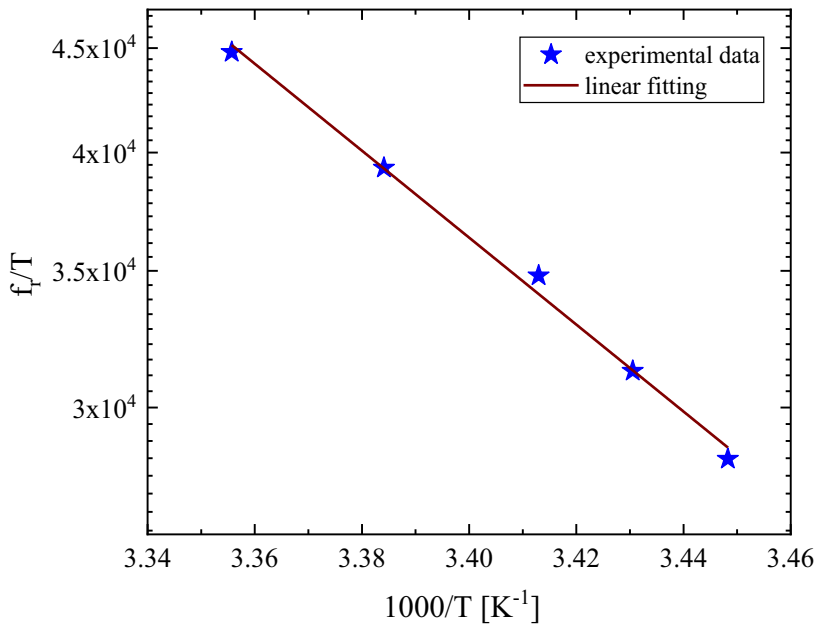

Fig. 7 Plot of the ratio $\left(f_{r} / T\right)$ as a function of the reciprocal temperature for DSP aqueous solutions with concentration $C=3.87 \mathrm{mM}$. The activation enthalpy $\Delta H^{*}$ required for aggregation mechanism is obtained from the slope of the linear graph and found equal to $\Delta H^{*}=4.23 \pm 0.34 \mathrm{kcal} / \mathrm{mol}$

concentration range studied here. It seems that $r<<1$ as expected for the majority of thermal relaxations in chemical reactions perturbated by the application of a compressional wave. In the case where $r<<1$, the following equation holds (Lamb 1965):

$\frac{2 \mu_{\max }}{\pi} \frac{C_{p}}{(\gamma-1)} \approx R\left(\frac{\Delta \mathrm{H}^{0}}{R T}\right)^{2} \exp \left(-\frac{\Delta \mathrm{H}^{0}}{R T}\right)$

where $C_{P}$ is the specific heat under constant pressure and $\gamma$ is the ratio $C_{P} / C_{V}$. The rest of the symbols have their usual meanings. $\Delta H^{0}$ is the difference enthalpy that can be directly estimated from the acoustic data through the temperature dependence of the relaxation amplitude. Since the values of $C_{P}$ and $\gamma$ are not known for the DSP aqueous solutions in the temperature range studied here, we will use the two following equations in order to be able to estimate the $\Delta H^{0}$ value (Lamb 1965):

$u^{2}=\frac{(\gamma-1) J C_{p}}{\theta^{2} \mathrm{~T}}$

where $\theta$ is the thermal expansion coefficient and $J=4.187$ Joule/cal is the Joule to calory conversion factor. Furthermore, we have:

$\frac{T^{2} C_{p} \mu_{\max }}{(\gamma-1)}=\left(\frac{T^{2} J}{u^{2}}\right)\left(\frac{C_{p}}{\theta}\right)^{2} \mu_{\max }$

By combining the last three equations and since the ratio $\left(\frac{C_{p}}{\theta}\right)^{2}$ is not temperature dependent in the narrow temperature range studied here, we are able to estimate the 
enthalpy difference $\Delta H^{0}$ from the slope and intercept of the $\ln \left(T \mu_{\max } / u^{2}\right)-1 / T$ plot, which is presented in Fig. 8.

The difference of enthalpy was found equal to $\Delta H^{0}=6.62 \pm 0.14 \mathrm{kcal} / \mathrm{mol}$. A criterion for the validity of the calculation is to check whether the condition $\Delta H^{0}>2.4 R T$ holds (Lamb 1965). Indeed, in our case this condition is fulfilled and our calculation is valid. The fact that $\Delta H^{0}$ was assumed independent of temperature in the

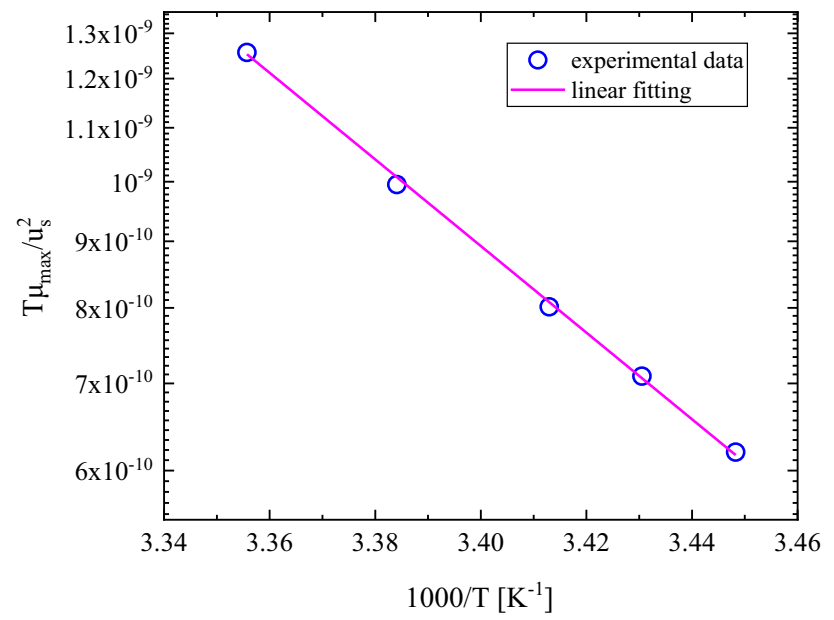

Fig. 8 Plot of $\ln \left(T \mu_{\max } / u^{2}\right)$ versus $1 / T$ for DSP aqueous solutions with concentration $C=3.87 \mathrm{mM}$. The enthalpy difference $\Delta H^{0}$ between the two states of the reaction is estimated from the slope and found equal to $\Delta H^{0}=6.62 \pm 0.14 \mathrm{kcal} / \mathrm{mol}$ temperature range studied here is further supported by the observed linearity of the $\ln \left(T \mu_{\max } / u^{2}\right)-1 / T$ plot. Semiempirical PM6 D3 method was used to calculate theoretically the enthalpy difference for the mechanism of Eq. (1). Recently it has clearly shown that other semiempirical quantum-chemical (SQC) methods, such as the OMx and OMx-Dn methods can be recommended in most cases for $\mathrm{C}, \mathrm{H}, \mathrm{N}, \mathrm{O}$, and $\mathrm{F}$ elements. Nevertheless, PM6 remain a valuable alternative for molecules containing other elements, such as P (Dral et al. 2016). The calculated value was found $\Delta H^{0}{ }_{t h}=6.56 \mathrm{kcal} / \mathrm{mol}$, which is close to the experimental value $\left(\Delta H^{0}=6.62 \pm 0.14 \mathrm{kcal} / \mathrm{mol}\right)$. These values are slightly different probably due to the fact that the calculation was performed in a vacuum environment without any interactions. The energy diagram for the DSP self-aggregation reaction is presented in Fig. 9. The two configurations shown are the monomer and dimer species and correspond to optimized structures. The calculations revealed that the aggregate is the most thermodynamically stable. The energy barrier $\Delta E$ shown in Fig. 9 can be estimated as the sum of the activation enthalpy $\Delta H^{*}$ and the difference of enthalpy $\Delta H^{0}$ related to the self-association mechanism as $\Delta E=\Delta H^{*}+\Delta H^{0}=4.23+6.62=10.85 \pm 0$. $37 \mathrm{kcal} / \mathrm{mol}$. The error in $\Delta E$ value has been estimated by applying the standard error propagation procedure.
Fig. 9 Energy diagram for the self-association reaction and the two configurations (left: monomer, right: dimer) as predicted from the theoretical calculations. $\Delta H^{*}, \Delta H^{0}$ and $\Delta E$ are the activation enthalpy, the enthalpy difference and the total energy barrier equal to $\Delta E=\Delta H^{*}+\Delta H^{0}$
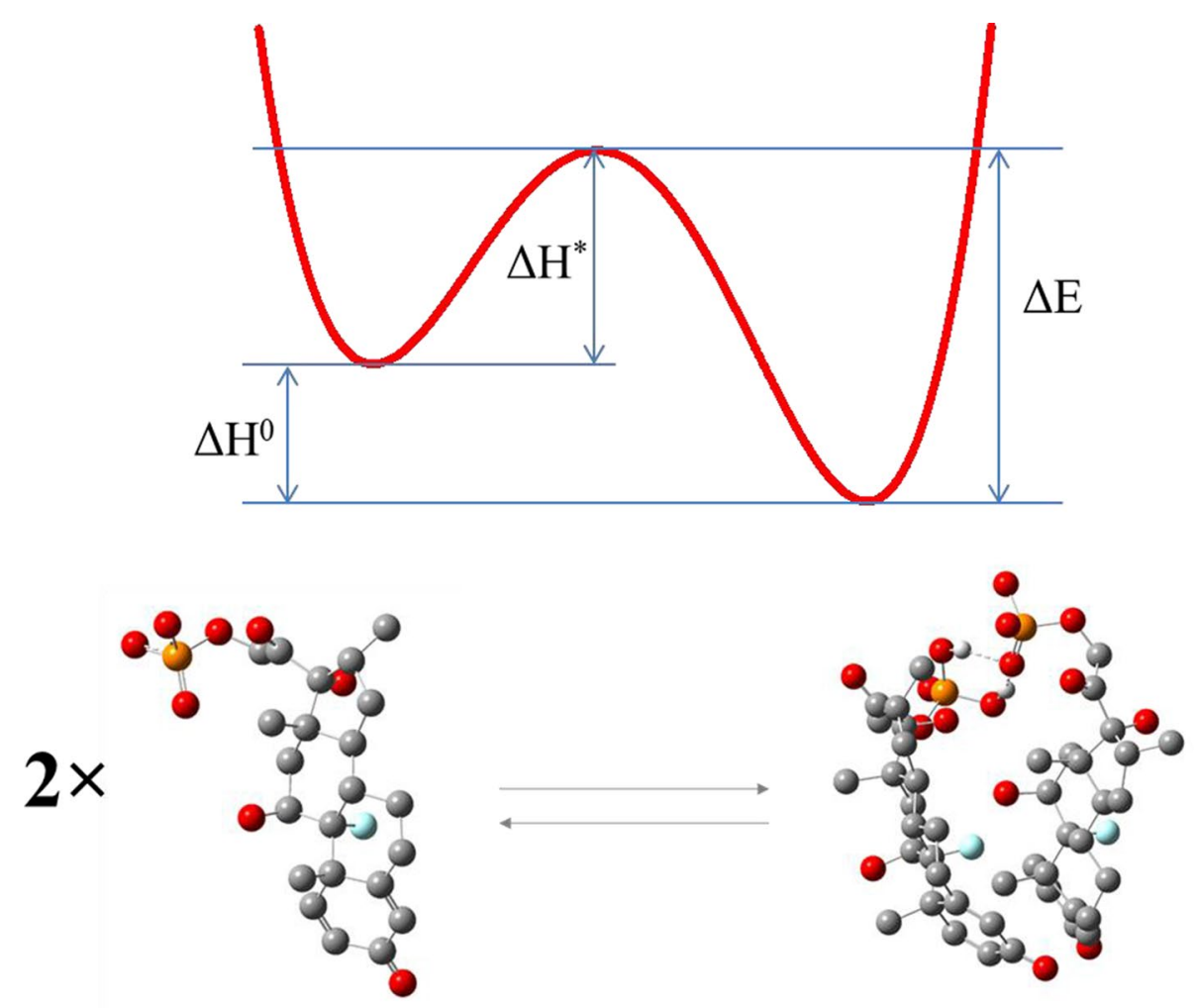


\section{Conclusions}

Aqueous dexamethasone sodium phosphate solutions were studied in the relatively dilute region by means of ultrasonic relaxation spectroscopy in the MHz frequency range. Temperature- and concentration-dependent measurements of the excess absorption coefficient were performed to elucidate the single relaxation observed in acoustic spectra, which is best fitted with a Debye-type function. The dominating process behind the relaxation is the self-association mechanism. The concentration dependence of the relaxation frequency and amplitude allowed us to exclude the presence of additional mechanisms in the frequency range studied. The concentration dependence of the specific conductivity exhibits an inflection point which is characteristic of self-aggregation process. The inflection point is observed at $2.82 \mathrm{mM}$, which is perfectly matches the concentration of the relaxation amplitude maximum. Analogous results have been observed for adiabatic compressibility further supporting the assignment of the experimentally detected relaxation to self-association. The aggregation number was expected to be low and experimentally determined equal to 2 . From the temperature dependence of the acoustic parameters, we were able to evaluate the thermodynamic characteristics of the self-association mechanism. The experimental activation enthalpy was found equal to $\Delta H^{*}=4.23 \pm 0.34 \mathrm{kcal} / \mathrm{mol}$, while the enthalpy difference equal to $\Delta H^{0}=6.62 \pm 0.14 \mathrm{kcal} / \mathrm{mol}$. The experimental results were compared with complementary theoretical calculations. The enthalpy difference was calculated theoretically by means of semiempirical PM6 D3 method equal to $\Delta H^{0}{ }_{t h}=6.56 \mathrm{kcal} / \mathrm{mol}$, which is close to the experimental value. Theoretical calculations revealed that the aggregate is the most thermodynamically stable. The theoretical molecular interaction study of the aggregation species revealed the formation of a stable aggregate composed of two monomers with binding energy of $2.91 \mathrm{kcal} /$ mol. The theoretical and experimental values of the volume change associated to the reaction further support our assumption that the present mechanism is related with the aggregation scheme.

Acknowledgements The authors gratefully acknowledge financial support from the University of Ioannina.

Author contributions CK: Investigation, Writing-review \& editing, PS: Investigation, Writing-review \& editing, AGK: Conceptualization, Investigation, Methodology, Supervision, Validation, Writingoriginal draft, Writing—review \& editing.

Funding This research did not receive any specific grant from funding agencies in the public, commercial, or not-for-profit sectors.

Availability of data Data are available from the corresponding author upon request.

\section{Declarations}

Conflict of interest On behalf of all authors, the corresponding author states that there is no conflict of interest.

\section{References}

Antignac JP, Le Bizec B, Monteau F, Andre F (2002) Differentiation of betamethasone and dexamethasone using liquid chromatography/ positive electrospray tandem mass spectrometry and multivariate statistical analysis. J Mass Spectrom 37:69-75. https://doi.org/ 10.1002/jms.260

Bae JR, Yi MH (2002) Ultrasonic velocity and absorption measurements in binary solutions of poly(acrylic acid) and in water solutions. J Korean Phys Soc 41:171-174

Bae JR, Yi MH, Lim JK (2001) Ultrasonic relaxation study in methyl acetate by using the high-Q ultrasonic resonator method. J Korean Phys Soc 38:670-673

Barfield RN, Schneider WG (1959) Excess ultrasonic absorption in diethylamine-water mixtures. J Chem Phys 31:488-494. https:// doi.org/10.1063/1.1730381

Becke A (1993) Density-functional thermochemistry. III. The role of exact exchange. J Chem Phys 98:5648-5652. https://doi.org/10. $1063 / 1.464913$

Bernasconi CF (1976) Relaxation kinetics. Academic Press, New York

Blandamer MJ (1973) Introduction to chemical ultrasonics. Academic Press, New-York

Cassileth PA, Lusk EJ, Torri S, DiNubile N, Gerson SL (1983) Antiemetic efficacy of dexamethasone therapy in patients receiving cancer chemotherapy. Arch Intern Med 143:1347-1349. https://doi.org/10.1001/archinte.1983.00350070063012

Cohen SP, Bicket MC, Jamison D, Wilkinson I, Rathmell JP (2013) Epidural steroids: a comprehensive, evidence-based review. Reg Anesth Pain Med 38:175-200. https://doi.org/10.1097/AAP.0b013 e31828ea086

Dral PO, Wu X, Spörkel L, Koslowski A, Thiel W (2016) Semiempirical quantum-chemical orthogonalization-corrected methods: benchmarks for ground-state properties. J Chem Theory Comput 12:1097-1120. https://doi.org/10.1021/acs.jctc.5b01047

Ensminger D, Bond LJ (2011) Ultrasonics: fundamentals, technologies, and applications. CRC Press, New York

Frisch MJ, Trucks GW, Schlegel HB et al (2013) Gaussian 09, Revision D.01, Gaussian, Inc., Wallingford, CT

Grunwald HW, Rosner F (1984) Dexamethasone as an antiemetic during cancer chemotherapy. Ann Intern Med 101:398. https://doi. org/10.7326/0003-4819-101-3-398_1

Herzfeld KF, Litovitz TA (1959) Absorption and dispersion of ultrasonic waves. Academic Press, New York

Huang H, Nishikawa S, Dong S (1999) Kinetic isotope effects of proton transfer reaction for amines by ultrasonic relaxation methods: unexpected evidence from the results in ethylamine solutions. $\mathrm{J}$ Phys Chem A 103:8799-8802. https://doi.org/10.1021/jp991223i

Kaatze U, Hushcha TO, Eggers F (2000) Ultrasonic broadband spectrometry of liquids a research tool in pure and applied chemistry and chemical physics. J Solution Chem 29:299-368. https://doi. org/10.1023/A:1005106618493

Kalampounias AG (2020) Exploring conformational change profile of n-propyl ester of formic acid by combining ultrasonic relaxation spectroscopy and molecular orbital calculations. J Mol Struct 1212:128146. https://doi.org/10.1016/j.molstruc.2020.128146

Kalampounias AG, Tsilomelekis G, Boghosian S (2013) Liquid phase dynamics of molten $\mathrm{M} 2 \mathrm{~S} 2 \mathrm{O} 7(\mathrm{M}=\mathrm{K}, \mathrm{Cs})$ : a temperature 
dependent Raman spectroscopic study. Vibr Spectr 65:66-73. https://doi.org/10.1016/j.vibspec.2012.11.021

Kouderis C, Siafarika P, Kalampounias AG (2021) Disentangling proton-transfer and segmental motion relaxations in poly-vinylalcohol aqueous solutions by means of ultrasonic relaxation spectroscopy. Polymer 217:123479. https://doi.org/10.1016/j.polym er.2021.123479

Lamb J (1965) Thermal relaxation in liquids, In: Physical Acoustics, Vol. 2, Part A, pp 203-280

Lee C, Yang W, Parr RG (1988) Development of the Colle-Salvetti correlation-energy formula into a functional of the electron density. Phys Rev B 37:785-789. https://doi.org/10.1103/PhysRevB. 37.785

Lee YH, Kwon HC, Kim JM (2017) Ultrasonic spectroscopy: applications in condensed matter physics and materials science. Cambridge University Press, Cambridge

Mpourazanis P, Stogiannidis G, Tsigoias S, Papatheodorou GN, Kalampounias AG (2019) Ionic to covalent glass network transition: effects on elastic and vibrational properties according to ultrasonic echography and Raman spectroscopy. J Phys Chem Solids 125:43-50. https://doi.org/10.1016/j.jpcs.2018.10.010

National Institutes of Health, National Heart, Lung, and Blood Institute (1995) Global initiative for asthma: global strategy for asthma management and prevention NHLBI/WHO Workshop Report. Bethesda, MD: National Institutes of Health. Jan: 77-100. NIH/ NHLBI Publication No. 95-3659

Nishikawa S, Gouhara R (1996) Ultrasonic relaxations in aqueous solutions of piperidine and pyrrolidine. Bull Chem Soc Jpn 69:18551861. https://doi.org/10.1246/bcsj.69.1855

Nishikawa S, Kamimura E (2011) Dynamic characteristic of amitriptyline in water by ultrasonic relaxation method and molecular orbital calculation. J Phys Chem A 115:535-539. https://doi.org/ 10.1021/jp1107729

Nishikawa S, Yasunaga T (1973) The kinetics of association-dissociation reaction in an aqueous solution of n-butylamine by means of ultrasonic absorption. Bull Chem Soc Jpn 46:1098-1101. https:// doi.org/10.1246/bcsj.46.1098

Nishikawa S, Haraguchi H, Fukuyama Y (1991) Effect of ether oxygen on proton transfer and aggregation reactions of amines in water by ultrasonic absorption method. Bull Chem Soc Jpn 64:1274-1282. https://doi.org/10.1246/bcsj.64.1274

Nishikawa S, Yasunaga T, Takahashi K (1973) Kinetic studies of fast reactions in aqueous solutions of amylamine by means of ultrasonic absorption. Bull Chem Soc Jpn 46:2992-2997. https://doi. org/10.1246/bcsj.46.2992

Petrucci S (1971) Ionic interactions, vol II. Academic Press, New York
Raynor JW, Minor W, Chruszcz M (2007) Dexamethasone at $119 \mathrm{~K}$. Acta Crystallogr E Struct Rep Online 63:o2791-o2793. https:// doi.org/10.1107/S1600536807020806

Roila F, Ballatori E, Ruggeri B, DeAngelis V (2000) Dexamethasone alone or in combination with ondansetron for the prevention of delayed nausea and vomiting induced by chemotherapy. N Engl $\mathrm{J}$ Med 342:1554-1559. https://doi.org/10.1056/NEJM20000525342 2102

Siafarika P, Kouderis C, Kalampounias AG (2021) Non-Debye segmental relaxation of poly- $\mathrm{N}$-vinylcarbazole in dilute solution. Mol Phys 119:e1802075. https://doi.org/10.1080/00268976.2020. 1802075

Sterne JA, Murthy S, Diaz JV, Slutsky AS, Villar J, Angus DC et al (2020) Association between administration of systemic corticosteroids and mortality among critically Ill patients with COVID19A meta-analysis. JAMA 324:1330-1341. https://doi.org/10. 1001/jama.2020.17023

Stogiannidis G, Tsigoias S, Kalampounias AG (2020) Conformational energy barriers in methyl acetate-Ethanol solutions: a temperature-dependent ultrasonic relaxation study and molecular orbital calculations. J Mol Liq 302:112519. https://doi.org/10.1016/j. molliq.2020.112519

Tamm K (1961) Encyclopedia of physics, Vol. 11, part I. Springer, New York

Tsigoias S, Kouderis C, Mylona-Kosmas A, Boghosian S, Kalampounias AG (2020) Proton-transfer in 1,1,3,3 tetramethyl guanidine by means of ultrasonic relaxation and Raman spectroscopies and molecular orbital calculations. Spectrochim Acta A 229:117958. https://doi.org/10.1016/j.saa.2019.117958

Tsigoias S, Papanikolaou MG, Kabanos TA, Kalampounias AG (2021) Structure and dynamics of aqueous Norspermidine solutions: an in-situ ultrasonic relaxation spectroscopic study, submitted

Verall RE, Nomura H (1977) Ion association and ultrasonic relaxation study of tetraalkylammonium and bis-tetraalkylammonium electrolytes in 2-propanol as a function of temperature and concentration. J Solut Chem 6:541-558. https://doi.org/10.1007/BF006 45419

World Health Organization (2019) World Health Organization model list of essential medicines: 21st list 2019. World Health Organization, Geneva

Publisher's Note Springer Nature remains neutral with regard to jurisdictional claims in published maps and institutional affiliations.

\section{Authors and Affiliations}

\section{Kouderis ${ }^{1}$ · P. Siafarika ${ }^{1}$ - A. G. Kalampounias ${ }^{1,2}$}

A. G. Kalampounias

akalamp@uoi.gr

1 Department of Chemistry, University of Ioannina, 45110 Ioannina, Greece
Institute of Materials Science and Computing, University Research Center of Ioannina (URCI), Ioannina, Greece 